\title{
„REBELL/IN/NEN (NICHT NUR) DES WORTES“_ MIGRATIONSGESTÜTZTE LITERARISCHE AUFBRÜCHE IN EIN POSTNATIONALES ÖSTERREICH. VON HADZIBEGANOVIC ZU INSAYIF UND MAANI
}

\begin{abstract}
Als Anhang zu ihrer Preisrede im Zuge der ersten Verleihung des österreichischen Literatur(förder)preises Schreiben zwischen den Kulturen (1997) hat die aus Brčko geborene Alma Hadzibeganovic, ein eigenwilliges, ihr Schreibverständnis erläuterndes Alphabet vorgelegt. Unter dem Buchstaben , $R^{\prime}$ hat sie den rebellischen Charakter des Beitrages dieser Gruppe (damals) junger Autorinnen und Autoren mit Migrationshintergrund selbstbewusst sichtbar zu machen versucht. Ein Blick auf die literarische Produktion der letzten 20 Jahre wird diese These bzw. diese Selbstpositionierung vielleicht nicht als die zentrale Entwicklungsperspektive in der neueren österreichischen Gegenwartsliteratur ausweisen, aber zugleich doch als eine ernst zu nehmende und in vielfältiger Weise das Erscheinungsbild mitprägende. Der Beitrag geht der Frage nach, wo und wie sich migrationsgestütztes Schreiben positioniert, und in welcher Weise Querverbindungen zu einer spezifischen österr. Tradition sprachkritisch-experimentellen Schreibens und Denkens anzutreffen sind, das per se national-sprachliche Begrenzungen unterläuft bzw. ironisch kommentiert.
\end{abstract}

SCHLÜSSELWÖRTER: Migration, experimentell-ironische literarische Verfahrensweisen, Verbindungen zur österreichischen Tradition der Sprach-Kritik und Sprach-Schöpfung, Alma Hadzibeganovic, Semir Insayif, Sama Maani

\section{"REBELL/IN/EN (NICHT NUR) DES WORTES" - MIGRATION SUPPORTED LITERARY TAKE OFFS TO POSTNATIONAL OUTLINES OF AUSTRIA. FROM HADZIBEGANOVIC TO INSAYIF AND MAANI}

ABSTRACT: In the codicil to her prize speech on the occasion of the first award ceremony (1997) for Schreiben zwischen den Kulturen Alma Hadzibeganovic, born in the bosno-croatian crossborder city of Brčko, presented a quite idiosyncratic and at the same time explanatory alphabet. The letter , $\mathrm{R}^{\prime}$ hereby emphasized a specific rebellious character of most of her same aged authors coming from migration backgrounds. Looking back on twenty years of literary and cultural production we probably may assert that selfpositionings as expressed by Hadzibeganovic in the meantime have contributed to differentiate at one hand literary techniques of authors coming from other backgrounds and to es-

Primus-Heinz Kucher - Alpen-Adria-Universität Klagenfurt, Klagenfurt am Wörthersee, 
tablish at the other hand connexions and intersections with the specific Austrian tradition of critical, ironical and experimental approaches in writing and reflecting words and worlds.

KEYwORDS: migration, experimental-ironical literary approaches, intersections with Austrian tradition of reflection and re-foundation of language, Alma Hadzibeganovic, Semir Insayif, Sama Maani

\section{1}

Als 1997 der vom Volumen her eher bescheidene, aber von der Intention her umso wichtigere und richtungsanzeigende Literaturpreis >Schreiben zwischen den Kulturen< eingerichtet und erstmals vergeben wurde (Schwaiger 2016: 27-30), um einer kaum sichtbaren, aber doch gewichtiger werdenden Szene von jungen Autor_innen, die im Zuge der Migrationen seit Ende der 1980er Jahre nach Österreich gekommen sind, ein Mindestmaß an Öffentlichkeit, Aufmerksamkeit und Würdigung zukommen zu lassen, trat mit der ersten Preisträgerin Alma Hadzibeganovic eine in mehrfacher Weise ungewöhnliche Stimme in den Vordergrund. Im Unterschied zur ersten größeren Immigrationswelle, die in den 1970er Jahren bekanntlich unter dem Schlagwort >Gastarbeiter`aus der Türkei und Ex-Jugoslawien firmierte, in Österreich jedoch keine ,Gastarbeiterliteratur' wie in der Bundesrepublik hervorgebracht hat (Esselborn 1997; Chiellino 2000), höchstens vereinzelte zaghafte Versuche im Genre der autobiographischen und interkulturellen Aufzeichnungsbücher (Şerafettin Yildiz 1995) sowie ein wenig Lyrik (z. B. Kundeyt Surdum 1988, Unter einem geliehenen Himmel), präsentierte sich Alma Hadzibeganovic erfrischend modern, unkonventionell, mit einem Wort: vielversprechend.

Letzteres war angesichts ihres damaligen Lebensalters (geboren 1972, d. h. sie war bei ihrem Debüt gerade 25 Jahre alt) ebenso überraschend, wie ihre das Schreiben begleitenden existentiell-kulturellen Koordinaten und Erfahrungen. Aus der 19911992 heftig umkämpften bosnisch-kroatischen Grenzstadt Brčko kommend absolvierte Hadzibeganovic seit 1990 ein Studium in Sarajevo, das zwei Jahre lang im Würgegriff serbisch-bosnischer Truppen lag, Jahre, die hochdramatisch waren und zugleich erstaunlich distanziert im Text durchklingen, zumal sie vordergründig kaum Spuren einer Traumatisierung aufweisen. Erstaunlich ist dies im Kontext literarischer Aufarbeitungen von Gewalterfahrungen, deren Eindringlichkeit medial omnipräsent gewesen ist, auch deshalb, weil der zeitliche Abstand zu ihnen sehr kurz war und die räumliche Komponente, einer Lagererfahrung strukturell verwandt, erschwerend ins Gewicht fiel (Bronfen u. a. 1999 bzw. Kasper 2016: 22-55). Der prämierte Text versucht diese verbürgt hochdramatische Belagerungserfahrung in Sprache umzusetzen und bedient sich dabei nicht, wie erwartet werden könnte, eines realistisch-deskriptiven oder reflexiv-diaristischen Modus. Er inszeniert geradezu, auch visuell markiert, eine Mischform aus diesen Modi mit akzentuiert sprachexperimentellen Elementen, wie solche zwar gelegentlich auch schon vor ihr, z. B. durch Emine S. Özdamar, ver- 
wendet wurden, aber doch nicht gängige sprachästhetische Verfahrensmuster bilden. Sichtbar wird diese sprachbewusste Orientierung bereits im Titel: zz00m: 24 Std.mix 1 of me oder Penthesilea in Sarajevo (Hadzibeganovic 1997: 4). Ein Titel wie der vorliegende ist als paratextliche Exposition oder Ouvertüre für die Lesererwartung nicht unerheblich: er collagiert semantisch offene wie klar besetzte Bedeutungen zu einem in mehrfacher Hinsicht plurilingualen und polyvalenten Auftaktsatz. Im >zz00m: 24 Std.mix 1<werden wir an optophonetische Kompositionen oder an Lautgedichte zwischen Raoul Hausmann und Ernst Jandl denken können, im >of me< scheint ein Subjektbezug durch, der im nachfolgenden Text durch die Ich-Erzählung auch eingelöst wird; mit >Penthesilea< und \Sarajevo< wird eine gleichsam moderne Troja-Belagerung einschließlich des Widerstands einer Frauenfigur aufgerufen, aber auch eine Kleist-Reverenz eingearbeitet, die in einem nachfolgenden Absatz weiter ausgeführt wird (in Zitaten und Reflexionen über den Status einer Penthesilea im Kontext militärischer Auseinandersetzung). Der Text selbst, bestehend aus sechs durch sprachlich auffällig markierte Zwischentitel voneinander abgegrenzte und das Geschehen strukturierende Kapitel, versetzt uns in ein Zimmer, offenbar in einem Studentenwohnhaus in Sarajevo, in dem die Ich-Erzählerin und ihre Freundin (Melli) vordergründig den Film Basic Instinct „in der amerikanischen OV“ als „Sprachübung für ihr Studium“ ablaufen lassen und diesen kommentieren. Eigentlich geht es aber darum, soziale und kulturelle Dynamiken eines Zusammenlebens einer kleinen Gruppe auf engstem Raum auszuloten, d. h. unter den Bedingungen eines mehrfachen Belagerungszustandes, der auch psychische Komponenten wie Identität, kulturelle Bräuche und Beziehungsmuster einschließt, und diese mit fragmentartigen Wahrnehmungen der umliegenden bzw. der Außenwelt kurzzuschließen. Wahrnehmungen, die vom gefährlichen WC-Besuch, über Gespräche mit Nachbarinnen bis hin zu verstohlenen Blicken auf serbische Stellungen an einem gegenüberliegenden Berg reichen, aber auch Vorbereitungen für Vorsprachen bei Behörden einschließen. Die beiden jungen Frauen wollen nämlich aus Sarajevo heraus und müssen sich daher entsprechende Passierscheine auf einem sogenannten „Amt der Serben“ besorgen, was sie in Kontakt mit Macho-Polizisten bringt, aber auch ethnisch-kulturelle Reflexionen und Strategien auslöst: das taktische Zurückdrängen des bosnisch-muslimischen Aspekts (in habitueller Anlehnung an den zuvor gesehenen Film - „Melli setzt instinktiv ihr Basic ein“ (Hadzibeganovic 1997: 9) einerseits und in zaghaften Verweisen auf eine, zuvor quasi verschwiegene serbische Mutter andrerseits (Hadzibeganovic 1997: 11), d. h. auf ein Signal der Zugehörigkeit auch an die Ethnie, die Nation des >Feindes und somit auf eine gefährliche Aufspaltung des Selbst. Womit der Text natürlich nicht mehr nur eine kuriose Episode aus dem Leben zweier Studentinnen thematisiert, sondern unvermittelt auch eine politische, psychische sowie sprachkulturelle Dimension erhält, die sich u. a. darin ausdrückt, dass in bestimmten Situationen die sprachliche aber auch gestische Modellierung eines Ich, einer Identität für das Gegenüber, in einem Fall Polizisten und Beamte, in einem anderen Freunde, sich zu verändern bzw. anzupassen oder im Sinne von Homi K. Bhabha eine Strategie 
von Mimikry zur Anwendung zu bringen hat (Bhabha 1994; 2004: 126). Zu diesen Strategien gehören auch eine gleichermaßen genderkritische und genderironische Sprachverwendung bzw. Sprachreflexion im Kontakt mit der männer- und gewaltdominierten Außenwelt einerseits und diese Außenwelt kommentierende Passagen der Protagonistin, welche immer intensiver die Kleist'sche Penthesilea-Figur, gebrochen durch Hegel, dabei einbeziehen, z. B. unmittelbar vor einem Granatenangriff sich Achill mit glühenden Wangen imaginierend:

\footnotetext{
Am großen Tisch, angesichts des Themas von Gesellschaft und Geschichte im Werke Kleists, schlage ich mich in die tonnenweise angehäufte Tragik im Kriegsgebiet der Liebe. Bei ihm [Kleist, P.-H.K.] war die Banalität nur als Ironisierung präsent. Sogar der Hegelsche Gedanke vom Tragischen als dem Konflikt zweier miteinander unvereinbarer Prinzipien ist im Vergleich zu Kleist einfach, denn jede der Kleistschen Gestalten trägt in sich bereits den Zwiespalt zweier miteinander nicht zu versöhnender Gegebenheiten. (Hadzibeganovic 1997: 17)
}

Mitten in diese Reflexionen - ihre Freundin Melli hockt bereits unter dem Tisch geht es dann los, so suggeriert es zumindest der Text mit einer Reihe von Granat- und Gewehrfeuer imitierenden Lautkompositionen: „BBAAMM! TATA-TATA-TA - BBBAAMMM!““(Hadzibeganovic 1997: 17) - Lautkompositionen, die das Ich nicht sonderlich zu irritieren scheinen, dreht sie doch das Radio - wir erfahren sogar welches (Omladinski Radio Sarajevo) lauter, aus der zuerst ein Nirvana-Song ertönt, gefolgt von einer Karadzic-Gusle, die er selbst als Student komponiert haben soll und offenbar als bitter-ironischer Subtext zu seiner Schlächter-Mentalität eingeblendet wird und fungiert („Oj Radoje, Radoje / krenimo u gradove / da ubijemo one gadove“ - „Oj Radoje, Radoje / gehen wir hinunter / zu den Städten / um den Unrat / dort zu töten“ (Hadzibeganovic 1997: 19). Der Text selbst - aber das ist fast nebensächlich - klingt nicht nur offen aus, geht in den abschließenden Absätzen in ein assoziatives Stakkato-Panorama von konkreten, imaginierten, von inneren und äußeren Bildern über, er skizziert im Ansatz ein Sprach-Bild-Denken und Sprach-Bild-Schreiben, das gleichermaßen vom Alltagswahnsinn ständiger Bedrohung wie von jenem eines intellektuell-politischen Gedächtnisses und der Notwendigkeit, dafür eine Sprache zu finden, mitunter zu erfinden, geprägt wirkt. Vor diesem Hintergrund lese ich das dem Text beigegebene Grosse AlmaAlphabet' in dem eine sprachbiographische Neuvermessung mit kritischen wie ironischen Seitenblicken unternommen wird. Entsprechend aufschlussreich präsentieren sich die Erklärungen, die jedem Buchstaben des Alphabets beigefügt sind, von A, das mit „Alma“ als „ambivalente Persönlichkeit“ (Hadzibeganovic 1997: 27), als die sich das Ich begreift, programmatisch umschrieben wird über I (für Integration, allerdings mit drei Fragezeichen versehen und das bereits 1997) bis hin zu dem wohl aufschlussreichsten konzeptuellen Vorschlag, nämlich das R betreffend, das bei Hadzibeganovic für „Rebellin“ steht und zwar für: „schonungslose Rebellin des Wortes“ (Hadzibeganovic 1997: 33). Diese Auto-Definition wird verknüpft mit der Perspektive, dass es auch jener Generation, d. h. jener der Migrantinnen und Migranten, zusteht, der 
Sprache, in der sie (auch) leben und schreiben, ihren spezifischen Wahrnehmungshorizont bzw. Stempel aufzudrücken. Es geht also nicht mehr nur darum, Integration mit einem mimetisch-assimilatorischen Sprachkonzept zu verknüpfen - je perfekter die Teilnahme an der Sprachgemeinschaft, desto integrierter bzw. höher der Anspruch auf Teilnahme an Integrationsprozessen - sondern darum, diese auch mitgestalten zu wollen im Wissen, dass sich das kulturelle Potential dieser Gruppen auch in der Sprache produktiv abbilden kann und soll. Zieht man für Österreich die Daten der Statistik Austria 2017 heran, so weisen darin immerhin 1,89 von 8,6 Millionen Menschen, also rund 22\%, einen ( $\mathrm{Im}$ )Migrationshintergrund auf, davon 1,4 Millionen der ersten Generation (migration \& integration 2017: 22). Daten, die eine klare Richtung anzeigen: ein Aufsprengen national-kultureller Homogenität, die Infragestellung bisher gültiger Grenzen und Parameter sowie die Perspektive einer postnationalen Realität (Vobruba 2012). Daher auch die graphisch durch Versalien besonders markierte Forderung: „Die Sprache entwickelt sich, und WIR VERÄNDERN SIE MIT. Schluß mit den schmierigen Aposteln der (sprachlichen) Starrheit.“ (Hadzibeganovic 1997: 33)

\section{2}

Alma Hadzibeganovic hat ihrem ersten Text wohl noch einen schmalen Band Kurzprosa ilda zuferka rettet die kunst (2000) und Das Stück (2007) folgen lassen, in dem der programmatische Anspruch auf Mit-Veränderung der Sprache, an produktiver wie subversiver Über-Schreibung zwar zurückgenommen erscheint, aber immerhin auch noch eigene Akzente setzt. Diese zeigen eine andere Facette sprachlicher Souveränität an, nämlich jene, den sprachlichen Alltagsjargon der Protagonistinnen, Migrantinnen aus Ex-Jugoslawien, auf die unter dem Label ,pop-Literatur' firmierende Tendenz einer sprachlichen Fokussierung auf die glitzernde Konsum- und Warenwelt abzustimmen. Bedauerlicherweise zog sie sich jedoch ab etwa 2007 aus der literarischen Produktion zunehmend zurück und ist nur noch gelegentlich als Mitwirkende (Drehbuch z. B.) an Filmprojekten in Erscheinung getreten.

Mit den Aktivitäten rund um den Literaturpreis `Schreiben zwischen den Kulturen<, der nach Hadzibeganovic einigen angehenden Schriftsteller_innen zu mehr und zu kontinuierlicher Aufmerksamkeit, ja in manchen Fällen zu einem Durchbruch als Autor_in verholfen hat (Dimitre Dinev, Seher Çakir, Julya Rabinowich, Anna Kim u. a. m.), sind auch erwähnenswerte Theaterprojekte und Auszeichnungen der >Wiener Wortstätte < auch hier bereits im Titel ein Signal - verknüpft. So hat z. B. im Jahr 2010 Olga Grjasnowa, die bereits 2007 am Literaturkurs im Rahmen des Ingeborg Bachmann-Preises in Klagenfurt teilgenommen hatte, dort noch vor ihrem Durchbruch als Romanautorin mit Der Russe ist einer, der Birken liebt (2012) ihr Stück Mitfühlende Deutsche aufgeführt und ausgezeichnet gesehen (Grjasnowa 2010: 95-107). In den letzten zehn Jahren hat sich dieses Spektrum bemerkenswert entwickelt und weiter ausdifferenziert, d. $h$. 
auch institutionell und verlegerisch verbreitern sowie im literaturöffentlichen Raum sich durchaus positionieren können, z. B. durch die Einrichtung eines Schwerpunktes Exil-Immigration in der Zeitschrift Zwischenwelt (Theodor Kramer Gesellschaft), der maßgeblich von Vladimir Vertlib protegiert wurde, im Veranstaltungsprogramm des Kulturzentrums Minoriten in Graz, der österreichischen Literaturhäuser insgesamt oder im Programm von bereits länger etablierten Verlagen wie z. B. Drava in Klagenfurt, Deuticke in Wien oder Haymon in Innsbruck, um nur einige Beispiele zu nennen. Inzwischen ist es auch durch literaturwissenschaftliche Studien, z. B. den Band Zeitenwende in den Fokus der österreichischen und internationalen Germanistik gerückt (Boehringer, Hochreiter, 2011; Babka, Cornejo, Vlasta, 2014; Bürger-Koftis, Pellegrino, Vlasta 2019).

Aus diesem Spektrum möchte ich im Folgenden zwei Autoren herausgreifen, die für meine Fragestellung von Interesse sind: Semier Insayif, einen in Wien 1965 geborenen Autor mit irakischem Anteil väterlicherseits, und Sama Maani, einen 1963 in Graz geborenen Autor und Psychoanalytiker mit iranischen Wurzeln, der seit Jänner 2017 auch einen Blog in der Tageszeitung Der Standard betreibt (mit Schwerpunkt auf Themen wie Islam-Wahrnehmung, Antisemitismus-Kritik, sexuelle Autonomie/ Befreiung, Narzissmus als Grundtendenz der Gegenwartskultur etc.) sowie auf Kateřina Černa (geboren 1985), eine Autorin mit tschechischem Familienhintergrund, die seit längerem in der Steiermark lebt, verweisen. So unterschiedlich die Werk-Biographien, die kulturellen Prägungen und die Themen ihrer Texte auch sein mögen, so verbindet sie doch zweierlei: eine jeweils akzentuierte Arbeit an bzw. in der Sprache mit der Intention, ihre sprachkritisch-reflexiven Zugänge auch mit sprach-politischen und ideologie-dekonstruktiven Haltungen bzw. Verfahren, und letzteres mitunter auch performativ begleitet, zu verknüpfen.

Gerade in dieser Hinsicht, d. h. der Verknüpfung des Sprach-Reflexiven mit dem Performativen, ist auf Insayif kurz einzugehen. Insayif ist zunächst vor allem als Lyriker seit Ende der 1990er Jahre hervorgetreten, der Debütband aus dem Jahr 1998, versehen mit einer $\mathrm{CD}$, trägt den Titel 69 konkrete annäherungsversuche; ihm folgten die beiden Lyrikbände Über Gänge verkörpert oder vom Verlegen der Bewegung in die Form der Körper (2001) sowie libellen tänze - blau pfeil platt bauch vier fleck (2004), wobei letztgenannter auf die sechs Cellosuiten von Johann Sebastian Bach zurückgreift und diese in eine eigenwillige Text-Textbild-Komposition umzusetzen unternimmt. Die jeweils sechszeiligen Textkompositionen werden nämlich einerseits in zwei Schriftgrößen und orthographischen Ausführungen auf eine Seite gesetzt. Am Seitengrund ist in kursiver Auszeichnung eine eigenwillige und poetische Tempoangabe beigegeben, die der in Partituren üblichen Tempo- und Rhythmusangabe (z. B. andante, adagio, allegretto etc.) und damit Interpretationshilfen ähnelt, wenn es z. B. zur ersten Suite heißt: „nah vor ort in allen farben wie zerstäubt". Andererseits werden sie, ausgenommen die erste Suite, beginnend mit einem prélude in sechs barocken Tanzformen (Allemande, Courante, Sarabande, Menuett, Bourrée, Gigue) durchvariiert. Das erste Gedicht fungiert zugleich als ,Stammgedicht', was vor allem bedeutet, dass die einzelnen Zeilen 
im jeweils ersten Vers des nachfolgenden Gedichts aufgegriffen werden (z. B. Vers drei als erste Zeile im dritten Gedicht etc.), woraus sich eine gleichermaßen strenge wie spielerisch wirkende Form ergibt. Das musikalische Neuarrangement erfolgte dabei durch den Cellisten Martin Hornstein. Martin Kubaczek hat dies in einer Besprechung entsprechend gewürdigt:

[...] ein durchstrukturiertes Projekt: Insayif kondensierte vorerst die Atmosphäre der Bachschen Solostücke in einem Ursprungsgedicht. Die Musik wird ihm so Materie, „Mutterstoff“, er subtrahiert daraus eine Grundform, deren phonetisches, assoziatives und metaphorisches Potential nun ausgereizt, variiert und permutiert wird. (Kubaczek 2005)

Diese als gelungen bezeichnete ,„̈sthetische Gratwanderung“ fungiert auch als eines der wenigen Beispiele zeitgenössischer Literatur von Schriftstellern mit Migrationshintergrund in einer repräsentativen Literaturgeschichte (Zeyringer, Gollner 2012: 741f.).

Konstruktiv eingesetzt wird also - wie bei Bach selbst - die Variabilität und Wiederholung eines relativ schmalen Materials, insofern als die sechszeiligen, mittig gesetzten Gedichte, begleitet sind einerseits von einer klein gedruckten, darüber platzierten lautsprachlichen Material-Variante, andererseits von der am Seitengrund gesetzten Kursivzeile. Es kann daher von einem Crossover von Lyrik und Musik gesprochen werden, wie ihn Insayif nach diesem Band als „Stimmakrobat" in weiteren Variationen immer wieder und stets um andere Akzente verschoben, angepeilt hat, 2011 etwa ,auf den Spuren von Matthias Claudius und Franz Schubert". Auf diese Weise gelingt nicht nur eine originelle Aneignung von fremder Tradition, sondern auch deren produktive Verschränkung mit Gegenwärtigkeit über kulturelle Grenzen hinweg.

Auch sein bislang einziger Prosaband Faruq (2009), dem Untertitel nach ein Roman, ist in vielfacher Weise ein Crossover zwischen Erzählung, Reflexion, Sprache und Musik, ein „Sprach- und Klangteppich“, wie er in einer der wenigen bislang vorliegenden einlässlicheren Arbeiten bezeichnet worden ist. Darüber hinaus kann er auch insofern als ein sehr komplexer Text angesehen werden, als sich in ihm Erinnerung und Gegenwart, die Identität aufspaltend und rekonstruierend, beständig eines Prozesses der Selbstüberprüfung aussetzen (Babka 2014: 139).

Ein Zwiegespräch zwischen einem Ich und Du, so der Prolog und Romaneinsatz, das als solches nur zögerlich in Gang zu kommen scheint, schlägt dabei die rhythmische Grundsignatur an. Indiz dafür sind die wiederholten Aufforderungen, doch endlich zu sprechen zu beginnen bzw. Zeugnis zu geben und zu leben - Aufforderungen, die nachdrücklich in den entstehenden Text-Körper eingeschrieben werden und dabei alle seine Komponenten einbeziehen: die Atmung und die daran beteiligten Organe (Stimmbänder, Zunge, Nase etc.), die Sprechakte selbst und die damit intendierten Wir- 
kungen im Gegenüber. Oft interpunktionslos, parataktisch und zugleich im Rhythmus ansteigend bis an den Rand der Atemlosigkeit, ausgreifend und auf Resonanz hoffend, so führt sich diese Stimme und ihre Rede ein:

[...] ergreifend muss sie jedenfalls sein die rede, raffiniert, gefühlvoll und logisch aufgebaut [...] sie muss um sich greifen, in die herzen der zuhörerinnen und zuhörer fassen, sie packen und nicht mehr loslassen, bis alle herz- und hirnkammern vollkommen besetzt und verwirrt sind und nur noch verrückt spielen, einfach nur noch verrückt spielen können, so als könnten sie ihren ohren nicht trauen (Insayif 2009: 12).

Das Ich und sein Gegenüber stehen in einer besonderen Beziehung oder, besser gesagt, Verstrickung zueinander. Sprachlich kommt diese insofern zum Ausdruck, als das Ich, seiner Identität ungewiss - „,nehmen wir einmal an, ich wüsste, wer ich bin... “ (Insayif 2009: 16), woraus eine Reihe weiterer Annahmen und Hypothesen nachfolgen - wortreich dieses Gespräch mit einem vordergründig stumm bleibenden (oder mit projizierten möglichen Antworten überhäuften und daher nicht zur Rede kommenden) Gegenüber an sich zieht. Nahezu jede Frage wird in deren Antwortmöglichkeit vorweggenommen und nachfolgend wieder zu einer ,neu gestellten frage transformiert, die doch nur die illusionsmaschine einer wahrheit ist, die ihren schein geheim zu grabe trägt“" (Insayif 2009: 19).

Erst nach weiteren zwanzig Seiten etwa erfahren wir, dass dieses Ich, dessen Identität zuvor unermüdlich in Zweifel gezogen, als eine von denkbaren Versuchsanordnungen in den Raum gestellt, gegen Einwände und Vorschläge hin- und hergewendet wird, und in eine Aufbruchsbewegung, offensichtlich aus einer Ebene in ein zunächst nicht erkennbares Ungewisses, gestellt erscheint, jedenfalls „fort von dieser beklemmung von dieser beklemmenden gewissheit" (Insayif 2009: 28). Und es ist wieder die Sprache, die eine weitere wichtige Fährte legt: das Wort schurūq (Insayif 2009: 30), ein arabisches Wort: Sonnenaufgang, eine Zeitangabe, auch eine Richtung am Himmel, aber offenbar auch im Ich selbst „oder aus ihm heraus. Eine idee“ (Insayif 2009: 30). Nichts steht dagegen im Text, zumindest in diesem Kontext davon, was üblicherweise mit diesem arabischen Wort assoziiert wird: die Spanne nämlich, innerhalb derer das Morgengebet zu verrichten sei (wie einschlägige online-Worterklärungen nahezu ausnahmslos suggerieren), also eine religiöse, identitätsverbindende Praxis.

Vielmehr stellt der Text auf Bewegung ab, konkret physische, aber auch auf existentiell-intellektuelle, ein Gehen, bei dem es gilt, ,ein zeichen nach dem anderen auf die erde stempeln“, eine Erde, ein Boden, ,in erwartung, betreten zu werden“, was unmittelbar darauf meint: „, beschriftet, bezeichnet“ (Insayif 2009: 31), ein Gehen über und in eine Landschaft, das, dem Atemholen verwandt, grundlegende Koordinaten einer Existenz abschreitet und sie im Abschreiten neu beschriftet, neu ausmisst. Dem kontinuierlichen Abschreiten korrespondiert nun ein Text, in dem der Rhythmus des Atmens, Gehens, Hörens, des Wahrnehmens dieser Bewegungen in gewisser Weise abgebildet erscheint: in kurzen, elliptischen Sätzen oder Satzteilen, durchwegs in 
Kleinschrift, als gelte es, diese Bewegung auch im Textbild als ruhig dahinfließende deutlich zu markieren:

[...] in bewegung, erst links, dann rechts, dann wieder links und wieder rechts und so weiter $[\ldots]$ in zwingender abwechslung, zu sich selbst, bis zur linie dieses flusses, an ihm entlang, zu seiner linken. das fließen. in entgegengesetzter richtung. das fließen des wassers. seiner schritte. seines ganges. geräusche der sohlen... (Insayif 2009: 32f.)

Dieses Gehen als Abschreiten eines Lebens öffnet schließlich Zugänge zu offenbar Verschüttetem, zu Erinnerungen, die im Zuge dieser Bewegungen „kehrten wieder. nach und nach. kehrten zurück. in seinen schädel [...] zum glück. seine skizzen über geschehnisse, über viele seiner geschehnisse. außen wie innen." (Insayif 2009: 37f.) Mit dieser Wiederkehr erschließt sich das Weitergehen neue, dynamischere Dimensionen: So drängen sich die Erinnerungen an den Vater zwischen die Erinnerungen an die eigene Jugend, an die ersten Reisen mit den Eltern zu verstreuten Verwandten, in Schweden und Bagdad und Erinnerungen an Liebeserlebnisse mit einer Frau. „Die Intensität der Beziehung zu ihr" wird, so Andrea Grill in einer (der bedauerlicherweise wenigen) Besprechung, „,mit kuriosen Substantivschöpfungen, wie ,tiefrote lippenpolster" und „herzskelett“ beschrieben. Je mehr man aber davon liest, und je öfter sie doch immer ein wenig anders sind, desto charmanter werden sie." (Grill 2009) Mitunter auch anstrengender, aber oft auch instruktiver, etwa verschiedene Bedeutungs-Facetten arabischer Wörter betreffend, z. B. zum Wort ghazál (Frau, Geliebte, Mutter, das Wesen der Liebe an sich; Insayif 2009: 42), mitunter auch orientalischer in der Bilderflut und in Bezug auf die Form des Erzählens, die - wohl kaum zufällig gerade in Bagdad-Erinnerungen - zum Thema wird:

[...] in bab- al-sheich / einem der ältesten bezirke der stadt, wuchsen die geschichten innerhalb von fünf minuten. auf bäumen. auf sträuchern. in den himmel hinein. fielen auf die erde. ins fenster. in seine augen. hakten sich dort fest... (Insayif 2009: 45)

Es handelt sich dabei nicht, wie ein erster Blick vielleicht insinuiert, um eine nostalgische Projektion in eine nie gelebte Landschaft, ist doch die Erzählerfigur, wie an späterer Stelle klar wird, bereits in Wien geboren. Vielmehr wird ein Bagdad aus dem Erinnerungshorizont des Vaters aufgerufen, ein Bagdad, in dem die Religionen und damit eine der Wurzeln späterer Konflikte, noch problemlos nebeneinander ko-existierten und in das der in Wien zum Arzt ausgebildete Vater nicht mehr zurückkehren will. Das Gehen, Metapher auch für das Aufsuchen von Orten und Erinnerungen, amalgamiert ,seine Geschichte mit der Geschichte seines Vaters, die Geschichte des Suchenden mit der Geschichte eines inzwischen wieder, und wie wir am Ende zur Kenntnis nehmen müssen, rätselhaft Verschwundenen, mit dem sich im Gehen eine ganz eigene Geschichte, ein Austausch entwickelt. z. B. die Geschichte der Liebesbeziehung zu einem Mädchen aus Wien, eine Episode, in der auch - selten sonst im Roman - die konkrete historische Zeit 
en passant angesprochen wird - ,er sah sie [...] im votivpark. Im jahre sechsundfünfzig. “ (Insayif 2009: 123) Mit dieser aufblitzenden Erinnerung sind nicht nur berührende Emotionen verbunden wie arabische Liebesgedichte des Vaters an diese junge Frau und Mutter des Erzählers, wie bald zu entdecken ist, sondern auch, migrations- und kulturgeschichtlich interessant, Einblicke in diese uns heute kaum mehr zugängliche Welt einer ersten irakischen Emigration nach Wien in den späten 1950er Jahren - Einblicke auch in politische Fraktionierungen dieser vorwiegend studentischen Gruppen, in ihre Projekte und Utopien. Oder Erinnerungen an die väterliche Sehnsucht nach Pistazien und zwei in seiner Jugend in der gesamten arabischen Welt überaus populären Sängerinnen, Uum Kultum und Fairouz, Chiffren beide für Konturen arabisch-irakischer Heimat, die in Autofahrten und abgewetzten Cassetten als Geräuschkulisse auftönt, eine Heimat, in die zurückzukehren allerdings nicht mehr ratsam erschien (Insayif 2009: 157). Weiter gehen heißt auch irgendwann an der Gegenwart anstreifen, etwa über Telefonate mit Verwandten, die zunehmend von Terror-Anschlägen, von Gewalt berichten, von Folter und Mord - der Name Abu Ghraib (Insayif 2009: 169) fällt dabei auch - sowie vom bevorstehenden Ende des Großvaters, der den Vater dann doch zwingt, nach Bagdad zu fliegen, angsterfüllt, ahnend, dass es eine Reise ohne Wiederkehr sein könnte. Ein Finale, in dem der Text durch eine subtile Rhythmussteigerung, eine Elliptisierung des Sprechens gleichsam andeutet, dass es auf ein gewaltsames Ende hinausläuft, ohne dieses offen zu legen und somit auch eine politisch-moralische Ebene anklingen lässt, die zuvor nie ostentativ ins Spiel gebracht worden ist: durch die Verschränkung eines arabischen Regen-Gedichts mit einer Sterbeformel und dem lakonischen Befund: „mein vater war zwischen wien und bagdad verloren gegangen" (Insayif 2009: 179). Insayifs Text und sein Erzähler-Ich schreiben sich somit in die Geschichte eines unablässigen Gehens mit ungewissem Ankommen und potentiellem Sich Verlieren ein, das kulturelle wie nationale Zuordnungen relativiert, wenn nicht gar hinter sich lässt.

\section{4}

Vor wenigen Jahren, 2014, hat der 1963 in Graz aus einer iranischen Familie gebürtige und als Psychoanalytiker arbeitende Sama Maani seinen ersten Roman Ungläubig vorgelegt, dem 2015 ein Essayband mit dem provokanten Titel Respektverweigerung. Warum wir fremde Kulturen nicht respektieren sollten. Und die eigene auch nicht folgte sowie nach einem weiteren Erzählband 2018 der zweite Roman Teheran Wunderland. Der Vollständigkeit halber sei noch angemerkt, dass auch Maani seine literarischen Anfänge im Umfeld der edition exil und des Literaturpreises \Schreiben zwischen den Kulturen`genommen hatte, der ihm 2004 für die Erzählung Der Heiligenscheinorgasmus (Neuauflage 2016) zugesprochen worden war. Bereits aus manchen der hier genannten Titel leitet sich die Vermutung ab, es auch in diesem Fall mit einem sprachaufmerksamen, wenn nicht sprachpolitisch ausgerichteten Autor zu tun zu haben. 
Bei Ungläubig handelt es sich um einen in Briefform verfassten Text zwischen einem in Graz Medizin studierenden iranischen Studenten (Arasch Bastani) und seiner Grazer Psychoanalytikerin, die ihm freilich nie antwortet, weshalb von Beginn an eine geradezu absurde (Nicht)Kommunikationssituation das angedachte und anvisierte therapeutische Gespräch mitbestimmt. Ergänzt und begleitet wird dieser Briefwechsel von einem weiteren zwischen einem Freund des Protagonisten und einem sogenannten Zentralkomitee, einer nach der Chomeini-Revolution ins Ausland geflüchteten anarcho-politischen und religionskritischen mysteriösen Vereinigung, der auch Verwandte des Protagonisten angehört hätten. Dieses legt der Text nahe und zieht es oft und unmittelbar darauf wieder in Zweifel. Natürlich bildet die komplizierte Familiengeschichte einen kongenialen Rahmen für kulturkontrastive Perspektivierungen, die bei aller Distanz des Protagonisten zum Iran geradezu liebenswürdige Facetten einer Welt, insbesondere einer wenig bekannten lebensfreudigen Teheraner Welt, erinnernd und mit Witz gepaart, in diese Briefwechsel einschmuggeln. Teil der psychisch exponierten Verfasstheit jenes Arasch und zugleich ironische Brechungsmomente im Hinblick auf die Dramatik seiner Situation sind unter anderem einzelne Lektüren, die gefährliche Identifikationsangebote bereitstellen, allen voran E.T.A. Hoffmanns Meister Floh. Die (Lese)Begegnung mit der Dörtje Elverdink-Figur, die in der Folge zu einer Kolik führt und Arasch in ein Delirium stürzen lässt, entpuppt sich nämlich als Wunschprojektion einer realen Beziehung, denn er ,[...] begann in einer Art Trance Hanna zu rufen, immer wieder Hanna." (Maani 2014: 27) Aus der ergänzenden Korrespondenz seines Freundes mit dem Zentralkomitee erfahren wir, dass dieser in Graz lebende Student und Schriftsteller mittlerweile auch in Teheraner psychiatrischen und oppositionellen Kreisen zu einem ,Fall' geworden sei. So wird eine Therapiesitzung, in der zunächst Freuds Essay Der Witz und seine Beziehung zum Unbewussten mit der als „Witz“ empfundenen Revolution kurzgeschlossen wird, zu Arasch' Fall. Inzwischen auch im fernen Teheran als psychisch erkrankt geltend, wird er als Mitglied eines Künstlerkollektivs in einer Grazer Anstalt vorgestellt. Wenn daraufhin aus seinen Texten exemplarisch Passagen vorgelesen werden, rückübersetzt ins „Teheranische“, weil doch jener Schriftsteller „in Graz aufgewachsen“ sei und auf Deutsch schreibe (Maani 2014: 73), wiewohl der Text immer wieder Sprachbilder aus der Teheranischen Familiengeschichte in die viel blassere Grazer Alltagswelt transferiert, so spannt sich damit ein dialogischer Raum auf, in dem die Differenz zwischen den Kulturen und die Projektionen in die jeweils andere zu einem produktiven Vexierspiel anheben. Östliche Fabulierkunst und westliches Weltwissen (Fanta 2014) unterfüttern die kunstvoll vorangetriebenen Gesprächsund Briefarrangements, um sie zu einem subversiv-grotesken Clash of Civilizations zu amalgamieren, in dem orthodoxe marxistische Revolutionsmaximen auf religiösen Messianismus prallen und in einen zunehmend psychopathologischen Alltag, dem der Boden brüchig wird, einmünden. Dass die verquere Geschichte dieses Studenten nicht gut ausgehen kann, liegt auf der Hand; dass hier ein Autor seinen Migrationshintergrund weniger autobiographisch und schlüssig nachvollziehbar als pointiert ironisch-gebro- 
chen und sprachästhetisch verspielt gestaltet, soll allerdings nicht gegen den offenen Ausgang der Geschichte gewendet, sondern vielmehr als interessantes Signum einer das bloß Migrantische überwindenden Schreibpraxis gesehen werden.

Dass eine beständig zwischen Ironie und anarchischen Projektionen changierende Geschichte auch sprach- und kulturpolitisches Sprengpotenzial aufweisen kann, führt Maanis zweiter Roman Teheran Wunderland eindringlich vor. Der Schauplatz ist wieder auf einer weitgespannten Achse zwischen der Steiermark und dem Iran/Teheran verortet, zwischen einer eher langweiligen Provinz und einem faszinierenden Raum, in dem in gewisser Weise Weltgeschichte, nämlich das Ende einer über zweitausendjährigen, theokratisch eingefärbten Monarchie geschrieben wurde. Über dieses Ende und das nachfolgende problematische Experiment der Revolution halten drei aus Teheran stammende Brüder ein Tribunal ab, bei dem Phasen jener Aufbruchshoffnungen und deren klägliches, im Einzelfall traumatisches Scheitern aufgerollt werden. Systematischer als in Ungläubig rahmen und strukturieren neuerlich das Revolutionsnarrativ sowie der Versuch, gegen verordnete Revolutionsmoral ins Absurde ausufernde Therapiekonzepte ins Feld zu führen, den Text. Die erzählerische Autorität ist wieder eine höchst ungewisse (Messner 2018), verstehe sie ja die Sprache jenes Wunderlandes nicht oder kaum mehr - „Sarsamine Ajayeb sind die einzigen Worte der Sprache Teherans, die ich erinnere" (Maani 2018: 101), um trotzdem das Erzählte in wortmächtigen, frappierenden Bildern für den Leser auszubreiten. Jene Therapiekonzepte reichen von postpubertären Liebes- und Jahreszeitgedichten, von proletarischen Poesiefesten (Maani 2018: 22), deren emanzipatorische Texte vom Regime zu Repressionszwecken instrumentalisiert werden, über die Lektüre eines „berüchtigten Epos“ des persischen Dichters Antschenani aus dem 11. Jahrhundert, das der Erzähler mit der deutschsprachigen Titelvariante Der von der Frauenkrankheit Erfaßte versieht (Maani 2018: 32), bis hin zu Sigmund Freuds Drei Abhandlungen zur Sexualtheorie (Maani 2018: 62), die ihn schlussendlich in ein Umerziehungslager bringen (Maani 2018: 70ff.), wo eine dezidiert als „Feministin“ ausgegebene Professorin „Aspekte der Teheraner Tempel-Masturbation“ als Einstimmung auf nachfolgende „Mädchenweihen“, d. h. Geschlechtsumwandlungen, unterrichtet, um dem Problem der das System störenden homosexuellen Neigungen gegenzusteuern (Maani 2018: 83). Der Erzähler erblickt in seiner partiellen Teilnahme an diesem Umerziehungsprogramm eine Chance, seine Eltern zu schützen und seinen Entschluss reifen zu lassen, aus dem Land zu fliehen, womit die ironisch-grotesken Scharmützel mit einer fehlgeleiteten Revolution und ihrer religiös-dogmatischen Kapriolen am Ende in eine pragmatische Perspektive einmünden:

Ich hatte mich, um die Eltern vor den Repressalien der Faschisten zu schützen, bereit erklärt, beim Mädchenprojekt mitzumachen. Vielleicht hatten mich auch die Argumente der Professorin und Teheraner Feministin überzeugt, die Religion Teherans wäre in der Lage, sich selbst auszutricksen, und ich hatte die Teilnahme am Mädchenprojekt als meinen heroischen Beitrag betrachtet, das Problem der Männerliebe in Teheran zu lösen. [...] Ich beschloß, nach Hause zu fliehen und Teheran zusammen mit den Eltern zu verlassen. (Maani 2018: 97) 
Resümierend darf also festgehalten werden, dass migrationsgestütztes Schreiben sich in der österreichischen Gegenwartsliteratur der letzten zwanzig Jahre nicht nur selbstbewusst positionieren konnte, sondern immer wieder, in vertrackter bzw. subkutaner Beziehung zur langen Tradition sprachexperimentellen und subversiv-grotesken Sprechens sich zu positionieren scheint und zu spannenden Verfahrensweisen und Texten führt. Texte, die inzwischen den Nachweis erbracht haben, dass dieses längst integrale, wenngleich literaturwissenschaftlich oft noch marginalisierte und naturgemäß wenig markttaugliche Schreiben das Diktum vom „toten Kadaver“ (Abel, Zaimoglu 2006: 159f.) gerade durch diese spezifischen Signaturen wie sie seit Hadzibeganovic eingeführt und nach ihr von Rabinowich, Insayif und neuerdings eben auch Maani (nebst einigen anderen) ausdifferenziert wurden, doch widerlegt oder zumindest stark relativiert haben.

\section{Literatur}

Babka, A. (2014). „Wir haben ein land aus worten“- Semier Insayifs Roman Faruq. Aussiger Beiträge. Germanistische Schriftenreihe aus Forschung und Lehre 8. Wien: Praesens, 137-152.

Babka, A., Cornejo, R., Vlasta, S. (Hrsg.) (2014). Begegnungen und Bewegungen: Österreichische Literaturen. Aussiger Beiträge. Germanistische Schriftenreihe aus Forschung und Lehre 8. Wien: Praesens.

Bhabha, H.K. (2004). The Location of Culture (2. Aufl.). London, New York: Routledge.

Boehringer, M.\& Hochreiter, S. (Hrsg.) (2011). Zeitenwende. Österreichische Literatur seit dem Millenium 2000-2010. Wien: Praesens.

Bronfen, E., Erdle, B. \& Weigel, S. (1999). Trauma. Zwischen Psychoanalyse und kulturellen Deutungsmustern. Wien, Köln, Weimar: Böhlau.

Bürger-Koftis, M., Pellegrino, R. \& Vlasta, S. (Hrsg.) (2019). Wokommstduher? Inter-, Multi- und Transkulturalität im österreichischen Kontext. Wien: Praesens (im Druck).

Chiellino, G. (2000). Interkulturelle Literatur in Deutschland. Ein Handbuch. Stuttgart, Weimar: Metzler.

Esselborn, K. (1997). Von der Gastarbeiterliteratur zur Literatur der Interkulturalität. Zum Wandel des Blicks auf die Literatur kultureller Minderheiten in Deutschland. Jahrbuch Deutsch als Fremdsprache, Jg. 23, 47-62.

Fanta, W. (8. Oktober 2014). Sama Maani: Ungläubig. Abgerufen von www.literaturhaus.at/index. php?id=10365.

Grill, A. (22. September 2009). Semir Insayif: Faruq. Abgerufen von www.literaturhaus.at/index.php?id=7357. Grjasnowa, O. (2010). Mitfühlende Deutsche. In Ch. Stippinger (Hrsg.), Preistexte 10. Wien: edition exil. Hadzibeganovic, A. (1997). zz00m: 24 Std.mix 1 of me oder Penthesilea in Sarajevo. In Ch. Stippinger (Hrsg.), Schreiben zwischen den Kulturen. Eine Anthologie (S. 4-26). Wien: edition exil.

Insayif, S. (1998). 69 konkrete annäherungsversuche. Konkrete Texte und CD. Wien: Edition Doppelpunkt. Insayif, S. (2004). Libellen tänze. Gedichte nach den sechs suiten für violoncello solo von johann sebastian bach. Innsbruck: Haymon.

Insayif, S. (2009). Faruq. Roman. Innsbruck: Haymon.

Kasper, J. (2016). Der traumatisierte Raum: Insistenz, Inschrift, Montage bei Freud, Levi, Kertész, Sebald und Dante. Berlin, Boston: de Gruyter.

Kubaczek, M. (25. November 2019). Semier Insayif: libellen-tänze. Abgerufen von http://www.literaturhaus.at/index.php?id=1578. 
Maani, S. (2014). Ungläubig. Roman. Klagenfurt, Celovec: Drava.

Maani, S. (2018). Teheran Wunderland. Roman. Klagenfurt, Celovec: Drava.

Messner, E. (Dezember 2018). Iran Absurdistan. Sama Maanis ungemütlicher Kurzroman „Teheran Wunderland". Abgerufen von http://versorgerin.stwst.at/artikel/dec-6-2018-2030/iran-absurdistan.

migration \& integration. zahlen. daten. indikatoren 2017 (6. April 2019). Abgerufen von https://www. integrationsfonds.at/fileadmin/user_upload/Statistisches_Jahrbuch_migration_integration20172.pdf.

N.N. (8. April 2011). Semir Insayif der Stimmakrobat. Der Standard-Online. Abgerufen von https:// derstandard.at/1301874206215/Auftritt-Semier-Insayif-der-Stimmakrobat.

Schwaiger, S. (2016). Über die Schwelle. Literatur und Migration um das Kulturzentrum exil. Wien: Praesens.

Vobruba, G. (2012). Der postnationale Raum. Transformation von Souveränität und Grenzen in Europa. Weinheim, Basel: Beltz.

Zaimoğlu, F. \& Abel, J. (2006). „Migrationsliteratur ist ein toter Kadaver”. Ein Gespräch. Text + Kritik. Zeitschrift für Literatur. Literatur und Migration. Sonderband, 9, 159-166.

Zeyringer, K. \& Gollner, H. (Hrsg.) (2012). Eine Literaturgeschichte. Österreich seit 1650. Innsbruck, Wien: StudienVerlag. 